\title{
Subcellular Localization of a Novel Alternative Splicing of IIIG9 and Colocalization with PPP1gamma Isoforms
}

\author{
C. Sousa* ${ }^{1}$, A. P. Vintém ${ }^{1}$, M. Fardilha ${ }^{1}$, O. da Cruz e Silva ${ }^{2}$ and E. da Cruz e Silva ${ }^{1}$ \\ ${ }^{1}$ Signal Transduction Lab, CBC and Department of Biology, University of Aveiro, Campus de \\ Santiago, 3810-193 Aveiro, Portugal \\ ${ }^{2}$ Neurosciences Lab, CBC, University of Aveiro, Campus de Santiago, 3810-193 Aveiro, Portugal
}

\section{ABSTRACT}

In testis we find mainly PPP1gamma2 isoform. We hypothesize that in different cell types we can find different regulatory subunits that may constitute targets for therapeutics of diseases such as male infertility, cancer and Alzheimer's disease. We identified a novel alternative splicing isoform of IIIG9 in testis, a known regulator of PPP1, IIIG9sT, and the aim of this study was its further characterization. We used a specific antibody for IIIG9sT in order to characterize its localization in bovine sperm cells. We also transfected IIIG9sT-GFP construct in mouse spermatogonia cells (GC-1 cells) and we used specific antibodies for each PPP1 isoform for the colocalization studies. We observed them under a fluorescent microscope and a LSM and quantified a high co-localization with PPP1gamma1 and 2 isoforms.

\section{INTRODUCTION}

A common translation modification is protein phosphorylation/dephosphorylation; that is a reversible process that enables cells to respond to several different signals in a dynamic way.

In humans we can find at least 13 PPPs (protein serine/threonine phosphatases). However the diversity of members is increased by the existence of different isoforms, e.g. there are two gamma isoforms of PPP1 in humans, the most ubiquitous PPP, PPP1gamma1 and PPP1gamma2, codified by a single gene (PPP1CC). That is, the splicing machinery recognizes the exons with precision, removes the introns from the pre-mRNA molecule and ligates exons, caused by exon skipping or intron maintenance, will constitute a different protein [1]. PPP1gammal is ubiquity distributed in different human tissues; however gamma2 isoform is exclusive of testis and sperm.

PPP1 is regulated, at the subcellular level, by protein subunits, so we hypothesize that in different cell types we can find different regulators, and the ones exclusive of a tissue/cell type can be used for therapy in several diseases such as cancer, male infertility and Alzheimer's disease. In order to identify the regulatory subunits involved in the regulation of PPP1gamma2 we performed a yeast two-hybrid screen in a testis cDNA library [2]. We found a new splice variant, that seems to be exclusive of testis [3] of a known PPP1 regulator, IIIG9 [4], that we named IIIG9sT.

\section{MATERIAL AND METHODS}

Samples: Slides of bovine sperm, mouse spermatagonia fixed cells, GC-1 cell line (ATCC CRL2053) and GC-1 transfected cells with GFP construct (IIIG9sT-GFP). Cells were grown on glass coverslips coated with poly-ornithine (Sigma, Portugal) and fixed in 4\% paraformaldehyde and pure methanol (Sigma, Portugal). Antibody staining was performed as previously described [5]. Briefly, slides were incubated at room temperature with primary antibodies: anti-PP1gamma antibody (Covalab, UK) at 1:100, anti-gamma2 (G502) at 1:500 and anti-IIIG9sT (BioGenes Gmbh, 
Germany) at 1:50 dilution. After washing in PBS, Texas red-conjugated goat anti-rabbit secondary antibodies (Molecular Probes, USA) were added for $2 \mathrm{~h}$ at 1:300 dilution. DNA was counterstained with DAPI in the Vectorshield mounting media (Vector, USA).

Imaging: Epifluorescent microscopy and confocal laser scanning microscopy (CLSM) were used. For each sample, in CLSM, a stack of images was acquired, each in a slightly different focal plane, and a $3 \mathrm{D}$ representation of the sample was obtained. The microscopes used were a Zeiss CLSM 510 and an Olympus IX-81 epifluorecent microscope available at the University of Aveiro. The images in the stacks were acquired using a $60 \times$ objective. We used specific software in order to quantify colocalization analysing several different cells and slides.

\section{RESULTS AND DISCUSSION}

We characterized the expression of IIIG9sT in GC-1 cells as an even distribution in both nucleus and cytoplasm (Fig.1); however in sperm cells it is absent from the equatorial region of the head and
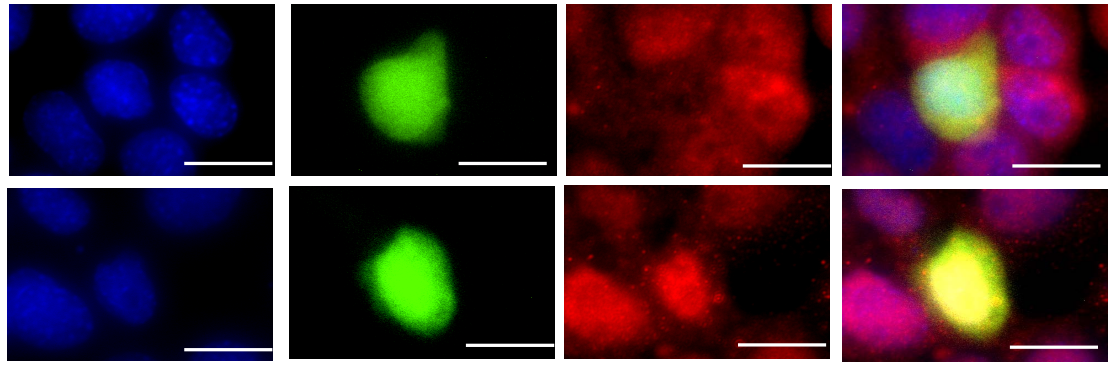

middle piece, being present in the tail and head (Fig.2). PPP1gamma2 has been described in sperm previously [5]. We found that PPP1alpha is absent both from these cells and sperm.

Fig. 1 - IIIG9sT-GFP in GC-1 cells (left to right): DAPI staining, IIIG9-GFP, Texas red conjugated secondary antibody + anti- -PPgammal (above) or 2 (bellow), overlay. (Scale bar $=20$ $\mu \mathrm{m})$

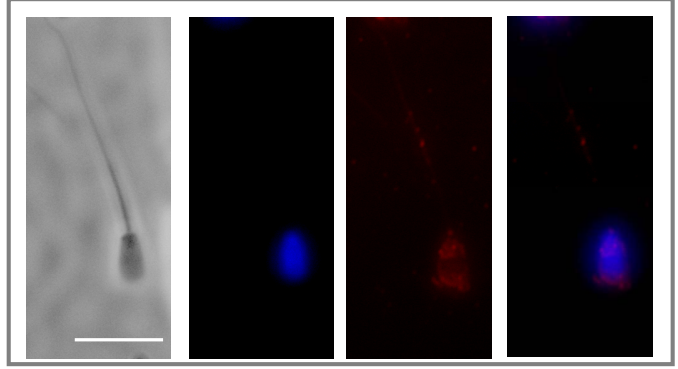

Table 1 - Overlap coefficient of sIIIG9-GFP with PPP1gammal and gamma 2 in GC-1 cells.

\begin{tabular}{|l|c|}
\hline & IIIG9sT \\
\hline PPP1gamma & $79.0 \%$ \\
\hline PPP1gamma2 & $86.0 \%$ \\
\hline
\end{tabular}

Fig. 2 - IIIG9sT in bovine sperm cells (left to right): PhC, DAPI staining, Texas red conjugated secondary antibody + anti-IIIG9sT, overlay $($ Scale bar $=20 \mu \mathrm{m})$.

So we analysed the colocalization of IIIG9sT with each PP1gamma isoform in GC-1 cells (Table1). We observed a very high overlap coefficient what indicates a high correlation of both distributions (of gamma1 and 2) with the one of IIIG9sT. 
CONCLUSIONS

We propose a model in which a tissue-specific PPP1 regulator (testis specific IIIG9 slice variant) may be used as target for therapeutic strategy of male infertility.

\section{ACKNOWLEDGMENTS}

This work was supported by FCT: CS received a PhD fellowship, and A.P.V. and M.F. received pos-doctoral fellowships.

\section{REFERENCES}

1. Wang, G. S. and Cooper, T. A., Splicing in disease: Disruption of the splicing code and the decoding machinery, Nat Rev Genet, 2007.

2. Fardilha, M. et al. Alternatively spliced protein variants as potential therapeutic targets for male infertility and contraception. Ann N Y Acad Sci 1030: 468-478, 2004.

3. Vintém, A.P. et al. Biochemical properties of an alternative splicing of IIIG9 found in testis, (manuscript in preparation).

4. Danielson, P. E. et al. Brain Res Gene Expr Patterns 1 (2): 83-88 (2002).

5. Sousa et al. Different subcellular distribution of novel regulators of protein phosphatase 1. Signal Transduction (submitted). 